OPEN ACCESS

Edited by:

Mauro Serafini,

University of Teramo, Italy

Reviewed by:

Wen Yen Juan,

United States Food and Drug

Administration, United States

Donato Angelino,

University of Teramo, Italy

*Correspondence:

Zongan Liang

2020324025198@stu.scu.edu.cn; liangza@scu.edu.cn

Ting Wang

w.t33@foxmail.com

†These authors have contributed equally to this work

Specialty section: This article was submitted to Nutritional Epidemiology,

a section of the journal

Frontiers in Nutrition

Received: 03 November 2021

Accepted: 14 January 2022

Published: 07 February 2022

Citation:

Lin P, Gui X, Liang Z and Wang T (2022) Association of Yogurt and

Dietary Supplements Containing

Probiotic Consumption With All-Cause and Cause-Specific Mortality in US Adults: A Population-Based Cohort

Study. Front. Nutr. 9:803076.

doi: 10.3389/fnut.2022.803076

\section{Association of Yogurt and Dietary Supplements Containing Probiotic Consumption With All-Cause and Cause-Specific Mortality in US Adults: A Population-Based Cohort Study}

\author{
Ping Lin ${ }^{1+}$, Xuezhen Gui ${ }^{2 \dagger}$, Zongan Liang ${ }^{1 *}$ and Ting Wang ${ }^{1 *}$ \\ ${ }^{1}$ Department of Respiratory and Critical Care Medicine, West China School of Medicine and West China Hospital, Sichuan \\ University, Chengdu, China, ${ }^{2}$ Division of Pulmonary Diseases, State Key Laboratory of Biotherapy of China, Sichuan \\ University, Chengdu, China
}

Background: Although probiotic intake had beneficial effects on several specific disorders, limited evidence was available about the benefits of probiotic intake in the general population. This study aimed to evaluate the relationship between yogurt (as a natural probiotic source) and dietary supplements containing probiotic consumption and mortality in US adults.

Methods: We conducted an observational cohort study comprised of a nationally representative sample of adults who were enrolled in the National Health and Nutrition Examination Survey (NHANES) between 1999 and 2014. Individuals were linked to the US National Death Index.

Results: We included 32,625 adults in our study. Of the study cohort, 3,539 participants had yogurt consumption, 213 had dietary supplements containing probiotic consumption, and the remaining participants $(28,873)$ did not have yogurt and/or dietary supplements containing probiotic consumption. During 266,432 person-years of follow-up, 3,881 deaths from any cause were ascertained, of which 651 were due to cardiovascular disorders and 863 were due to cancer. Weighted Cox proportional hazards models suggested that yogurt consumption was inversely associated with all-cause mortality (adjusted hazard ratio $(\mathrm{HR}), 0.83$ [95\% confidence interval $(\mathrm{Cl})$, 0.71-0.98]) but not cardiovascular mortality (adjusted HR, 0.68 [95\% Cl, 0.43-1.08]) and cancer mortality (adjusted HR, 1.00 [95\%Cl, 0.72-1.38]). However, dietary supplements containing probiotic were not associated with decreased all-cause and cause-specific mortality.

Conclusions: The present study suggested that yogurt consumption was associated with a lower risk of all-cause mortality among U.S. adults. Yogurt consumption in diet might be a sensible strategy for reducing the risk of death.

Keywords: probiotic, yogurt, NHANES, mortality, population 


\section{INTRODUCTION}

The human gastrointestinal tract harbors a complex community of microbes called the gut microbiota (1). The composition and metabolic activity of gut microbiota are now known to co-develop with the host beginning at birth and are under the influence of numerous factors (2). Gut microbiota and its disturbance have been associated with the pathogenesis of both intestinal and extra-intestinal disorders including gastroenterological diseases, metabolic disorders, cardiovascular diseases, autoimmune diseases, and neuropsychiatric disorders (3-7).

Probiotic intake might restore and maintain normal microbiota composition and function (8). Therefore, probiotic intake might have a positive impact on human health (9). Previous studies had suggested that probiotic might improve cognitive function and metabolic status (10), alleviate symptoms of various gastrointestinal disorders (11), decrease circulating levels of inflammatory biomarkers (12), improve glycemic and blood pressure control $(13,14)$, assist weight management in patients with type 2 diabetes (15), reduce antibiotic resistance (16), and prevent rhinovirus infections in preterm infants (17). Although published studies showed that probiotic intake had beneficial effects on people with specific disorders, limited evidence was available on the benefits of probiotic intake in the general population. Therefore, the recommendation to implement using of probiotic to provide health benefits in the general population needed further investigation (18).

In this study, we aimed to examine the relationship between yogurt (as a natural probiotic source) and dietary supplements containing probiotic consumption and mortality in a large cohort of participants in the National Health and Nutrition Examination Survey (NHANES), 1999-2014. We hypothesized that yogurt and dietary supplements containing probiotic consumption would be negatively associated with mortality.

\section{METHODS}

\section{Study Population}

The NHANES survey is a national program of studies aimed to evaluate the health and nutritional status of the noninstitutionalized US population using a complex, multistage, probability sampling design (19). The survey was conducted periodically since 1999 and a nationally representative sample of about 5,000 persons was examined each year. Data were collected by standardized in-person interviews and physical examinations.

In the present study, 82,091 participants from the NHANES surveys between 1999 and 2014 were included. All participants were linked to the US National Death Index (NDI), which provided mortality follow-up data through December 31, 2015 (20). We excluded participants aged $<18$ years at interviews $(n=34,812)$ and those with missing information on BMI ( $n$ $=3,232$ ), laboratory tests (white blood cell count, hemoglobin,

Abbreviations: NHANES, the National Health and Nutrition Examination Survey; NDI, the National Death Index; HR, hazard ratio; CI, confidence interval; BMI, body mass index. platelet count, total bilirubin, creatinine, and blood urea nitrogen) $(n=2,861)$, medical conditions (hypertension, diabetes, asthma, congestive heart failure, coronary heart disease, stroke, chronic bronchitis, and cancer) $(n=4,353)$, yogurt and dietary supplements containing probiotic consumption $(4,208)$. Therefore, a total of 32,625 participants remained in our cohort for analysis. Figure 1 presents a flowchart of participant selection.

\section{Assessments of Probiotic Intake}

In this study, probiotic intake was considered when a participant reported consumption of yogurt or dietary supplements containing probiotics $(21,22)$. We utilized the Dietary Interview-First Day (the 24-h dietary recall interview before the survey) and Dietary Interview-Second Day (the second 24-h dietary recall interview collected by telephone 3 to 10 days after the first interview) to assess yogurt consumption. We utilized the Dietary Supplement Use 30-Day (1999-2014), a questionnaire that collected personal interview data on food supplement use during a 30-day period before the interview date, to assess dietary supplements containing probiotic. The detailed probiotic consumption information is described in Supplementary Material 1.

\section{Statistical Analysis}

We analyzed the data using appropriate sampling weights (1/4*WTDR4YR for $1999-2002$ and $1 / 8^{*}$ WTDR2D for 20032014) to account for the complex survey design applied by the NHANES survey. We described baseline characteristics using percentages for categorical variables and means and standard deviations for continuous variables. We compared baseline characteristics using the Mantel-Haenszel $\chi^{2}$ test for categorical variables and the linear regression for continuous variables. We used weighted Cox proportional hazards models to calculate the hazard ratios (HRs) and corresponding $95 \%$ confidence intervals (CIs). Survey-weight adjusted multivariable Cox proportional model was adjusted for potential confounders that had been shown to be associated with mortality. Model 1 was not adjusted for any factors. Model 2 was adjusted for age, sex, race, and body mass index (BMI). Model 3 was adjusted for age, sex, race, BMI, white blood cell count, hemoglobin, platelet count, total bilirubin, creatinine, blood urea nitrogen, hypertension, diabetes, asthma congestive heart failure, coronary heart disease, stroke, chronic bronchitis, and cancer. Subgroup analyses were conducted by examining demographic characteristics, including age ( $<60 \mathrm{y}, \geq 60 \mathrm{y}$ ), sex (man, female), race/ethnicity (Mexican American, Other Hispanic, Non-Hispanic White, Non-Hispanic Black, Other Race), and BMI $\left(\mathrm{kg} / \mathrm{m}^{2}\right)(<18.5,18.5$ to $24.9,25$ to $29.9, \geq 30$ ). We used Stata version 14.0 (Stata Corp) and R version 3.6.3 (R Foundation for Statistical Computing) for statistical analysis and considered a two-tailed $p$-value less than 0.05 to be statistically significant.

\section{RESULTS}

\section{Participant Characteristics}

A total of 32,625 adults aged 18 years or older were included in this analysis. Of the study cohort, 3,539 participants had yogurt 


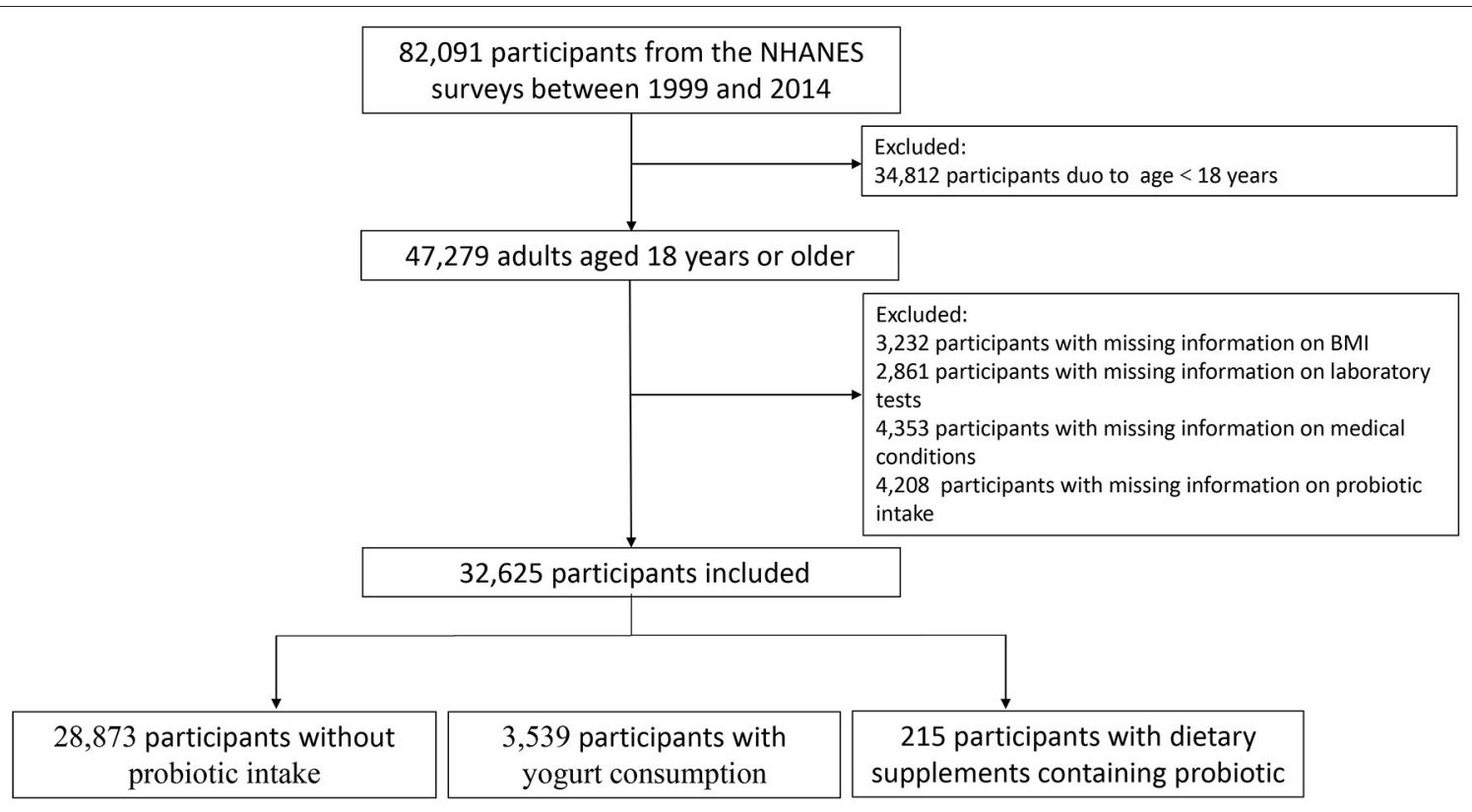

FIGURE 1 | Flowchart of patient selection.

consumption, 213 had dietary supplements containing probiotic consumption, and the remaining participants $(28,873)$ did not have yogurt and/or dietary supplements containing probiotic consumption. Characteristics of participants with different statuses of probiotic consumption were significantly different. Participants with yogurt or dietary supplements containing probiotic consumption were more likely to be older, to be a woman, to have low BMI, to be Non-Hispanic White, to have less hypertension, and to have cancer. The detailed baseline characteristics are shown in Table $\mathbf{1 .}$

\section{Associations Between Probiotic Exposure and Mortality}

During 266,432 person-years of follow-up, 3,881 deaths from any cause were ascertained, of which 651 were due to cardiovascular disorders and 863 were due to cancer. We evaluated associations of probiotic intake with mortality by logistic regression models with unadjusted and adjusted covariates (Table 2).

For unadjusted analyses (Model 1), yogurt consumption was not associated with a reduced risk of all-cause mortality (HR, 0.86 [95\%CI, 0.73-1.02]), cardiovascular mortality (HR, 0.66[95\%CI, 0.41-1.04]), and cancer mortality (HR, 1.03 [95\%CI, 0.751.41]). After adjusting for potential confounders (Model 2 and 3), yogurt consumption was inversely associated with all-cause mortality (HR, 0.83 [95\%CI, 0.71-0.98]) but not cardiovascular mortality (HR, 0.68 [95\%CI, 0.43-1.08]) and cancer mortality (HR, 1.00 [95\%CI, 0.72-1.38]) (Model 3).

However, dietary supplements containing probiotic were not associated with decreased all-cause and cause-specific mortality (Table 2). The unadjusted RRs of all-cause, cardiovascular, and cancer mortality were 0.64 (95\% CI $0.36,1.14), 0.83$ (95\% CI $0.25,2.72), 1.12$ (95\% CI $0.41,3.07)$, respectively. The adjusted
RRs of all-cause, cardiovascular, and cancer mortality were 0.74 (95\% CI 0.43, 1.29), 1.13 (95\% CI 0.34, 3.67), 1.34 (95\% CI 0.50, $3.62)$, respectively.

\section{Subgroup Analysis}

We further explored the association of yogurt consumption with all-cause mortality according to gender, age, race/ethnicity, and BMI (Table 3). We found that the association between yogurt consumption and decreased all-cause mortality was statistically significant among female, older participants (aged $\geq 60 \mathrm{y}$ ), and Non-Hispanic Black, while the association was weak and not statistically significant in other gender, ages, race/ethnicity, and weight subgroups.

\section{DISCUSSION}

In this large, nationally representative sample of US adults, we observed that participants with yogurt consumption had a lower risk for all-cause mortality compared with participants without yogurt consumption that persisted after adjustment for demographic characteristics, laboratory tests, and clinic comorbidities. In subsequent subgroup analyses, this yogurt-mortality inverse association was found to be present predominantly among female, older participants, and Non-Hispanic Black.

The association between yogurt consumption and mortality was inconsistent in previous studies. Schmid et al. reported that yogurt consumption was associated with a decreased risk of all-cause mortality (HR, 0.89 [95\%CI, 0.86-0.93]) in the Nurses' Health Study and The Health Professionals FollowUp Study, whereas Soedamah-Muthu et al. and Praagman et al. failed to discover a significant association between yogurt 
TABLE 1 | Participants baseline demographic and clinical characteristics.

\begin{tabular}{|c|c|c|c|c|}
\hline Characteristic & Without probiotic intake $(N=32,965)$ & Yogurt consumption $(N=3,539)$ & $\begin{array}{l}\text { Dietary supplements containing } \\
\text { probiotic }(N=213)\end{array}$ & $p$-value \\
\hline \multicolumn{5}{|l|}{ Baseline characteristics } \\
\hline Age (y) & $46.2 \pm 16.8$ & $48.3 \pm 16.4$ & $50.1 \pm 15.7$ & $<0.001$ \\
\hline Man (\%) & 49.7 & 35.4 & 27.3 & $<0.001$ \\
\hline $\mathrm{BMI}\left(\mathrm{kg} / \mathrm{m}^{2}\right)$ & $28.6 \pm 6.6$ & $27.7 \pm 6.4$ & $27.1 \pm 6.5$ & $<0.001$ \\
\hline Race (\%) & & & & $<0.001$ \\
\hline Mexican American & 8.4 & 6.0 & 2.6 & \\
\hline Other Hispanic & 5.1 & 4.2 & 5.2 & \\
\hline Non-Hispanic White & 69.3 & 79.0 & 81.7 & \\
\hline Non-Hispanic Black & 11.6 & 4.8 & 6.5 & \\
\hline Others & 5.5 & 6.0 & 3.9 & \\
\hline \multicolumn{5}{|l|}{ Laboratory tests } \\
\hline Hemoglobin (g/dL) & $14.4 \pm 1.5$ & $14.1 \pm 1.3$ & $14.2 \pm 1.1$ & $<0.001$ \\
\hline WBC (1,000 cells/uL) & $7.3 \pm 2.3$ & $7.1 \pm 2.4$ & $6.8 \pm 1.8$ & $<0.001$ \\
\hline Platelets ( 1,000 cells/uL) & $258.5 \pm 67.2$ & $252.5 \pm 65.4$ & $249.0 \pm 63.7$ & $<0.001$ \\
\hline Total bilirubin (umol/L) & $12.2 \pm 5.3$ & $12.5 \pm 5.1$ & $12.8 \pm 4.8$ & 0.0012 \\
\hline Creatinine (umol/L) & $78.1 \pm 33.2$ & $75.2 \pm 20.4$ & $75.1 \pm 16.8$ & $<0.001$ \\
\hline BUN (mmol/L) & $4.7 \pm 1.9$ & $4.8 \pm 1.7$ & $4.7 \pm 1.6$ & 0.0190 \\
\hline \multicolumn{5}{|l|}{ Comorbidities } \\
\hline Hypertension (\%) & 29.7 & 26.8 & 24.7 & 0.0002 \\
\hline Diabetes mellitus (\%) & 8.1 & 6.4 & 9.9 & 0.0002 \\
\hline CHD (\%) & 3.4 & 3.1 & 2.8 & 0.5734 \\
\hline $\mathrm{CHF}(\%)$ & 2.3 & 1.8 & 1.5 & 0.0707 \\
\hline Asthma (\%) & 13.4 & 12.5 & 18.7 & 0.0070 \\
\hline Stroke (\%) & 2.6 & 1.7 & 2.3 & 0.0014 \\
\hline Chronic bronchitis (\%) & 6.3 & 4.1 & 9.4 & $<0.001$ \\
\hline Cancer (\%) & 8.7 & 10.8 & 16.4 & $<0.001$ \\
\hline
\end{tabular}

$B M I$, body mass index; WBC, white blood cell; BUN, blood urea nitrogen; CAD, coronary heart disease; CHF, congestive heart failure.

Continuous variables were expressed as means and standard deviations and categorical variables were expressed as percentages. Means and percentages are weighted.

consumption and all-cause mortality in the Whitehall II cohort (HR, 0.74 [95\%CI, 0.53-1.05]) and the European Prospective Investigation into Cancer and Nutrition-Netherlands cohort (HR, 0.95 [95\%CI, 0.85-1.07]) $(23,24)$. The inconsistent findings might be due to different sociodemographic characteristics and follow-up times. In the present analysis, we observed that individuals with yogurt consumption had a $17 \%$ lower risk for all-cause mortality. This study was a national survey with a large sample size and a long follow-up (mean 8.1 years), which could offer reliable evidence and further reinforce earlier results. Moreover, we further conducted subgroup analyses by examining demographic characteristics (age, sex, race/ethnicity, and BMI). We found that the health benefits of yogurt consumption were particular among female, older participants (aged $\geq 60 \mathrm{y}$ ), and Non-Hispanic Black, indicating that these individuals were more likely to benefit from yogurt consumption. Unfortunately, the exact reasons for these observed demographic characteristics differences in the inverse associations were not clear. Sex-related differences might be due to the metabolism of sex hormones regulated by the gut microbiome (25).

We observed a reduction in mortality in yogurt consumption rather than dietary supplements containing probiotic in this study. Yogurt is a nutrient-dense fermented dairy product and is associated with high nutritional value (26). In addition to probiotic strains, yogurt is also a good source of abundant micronutrients and macronutrients, including calcium, Fe, $\mathrm{Mn}$, zinc, vitamins B-6/B-12, riboflavin, and protein $(27,28)$. These nutrients are essential for the sustenance of the functioning of the human body. Therefore, these nutrients might have a role in the beneficial effects of yogurt consumption compared to the supplementation of probiotic strains into a carrier liquid only. In addition, only a small number of people (about $0.65 \%$ ) who had a consumption of dietary supplements containing probiotic in diet might be responsible, at least in part, for these unexpected results.

Our results were supported by the existence of data showing a biological plausibility for the health benefits of probiotic. First, probiotic intake might be helpful to reduce risk factors for all-cause mortality and cardiovascular mortality such as obesity, hypertension, and type 2 diabetes (29-31). Second, probiotic foods had been reported to repress oxidative stress and inflammatory marker profile in both healthy persons and patients with disorders (32-34). Third, data also suggested that the use of probiotic might improve endothelial function and reduce arterial stiffness (35). Fourth, previous investigators found that 
TABLE 2 | Association of probiotic exposure with mortality in 32,625 participants of national health and nutrition examination survey, $1999-2014$.

\begin{tabular}{|c|c|c|c|c|c|c|}
\hline Exposure & Model $1^{\mathrm{a}}$ HR $(95 \% \mathrm{Cl})$ & $p$-value & Model $2^{\mathrm{b}}$ HR $(95 \% \mathrm{Cl})$ & $p$-value & Model $3^{c}$ HR $(95 \%$ Cl) & $p$-value \\
\hline \multicolumn{7}{|l|}{ All-cause mortality } \\
\hline Yogurt & $0.86(0.73,1.02)$ & 0.096 & $0.79(0.67,0.93)$ & 0.005 & $0.83(0.71,0.98)$ & 0.035 \\
\hline Probiotic supplements & $0.64(0.36,1.14)$ & 0.132 & $0.67(0.38,1.19)$ & 0.181 & $0.74(0.43,1.29)$ & 0.300 \\
\hline \multicolumn{7}{|c|}{ Cardiovascular mortality } \\
\hline Yogurt & $0.66(0.41,1.04)$ & 0.076 & $0.62(0.39,0.99)$ & 0.048 & $0.68(0.43,1.08)$ & 0.109 \\
\hline Probiotic supplements & $0.83(0.25,2.72)$ & 0.763 & $0.99(0.30,3.24)$ & 0.993 & $1.13(0.34,3.67)$ & 0.837 \\
\hline \multicolumn{7}{|l|}{ Cancer mortality } \\
\hline Yogurt & $1.03(0.75,1.41)$ & 0.845 & $0.98(0.71,1.35)$ & 0.904 & $1.00(0.72,1.38)$ & 0.972 \\
\hline Probiotic supplements & $1.12(0.41,3.07)$ & 0.815 & $1.21(0.45,3.27)$ & 0.699 & $1.34(0.50,3.62)$ & 0.555 \\
\hline
\end{tabular}

a Unadjusted.

${ }^{b}$ Adjusted for age, sex, race, and body mass index.

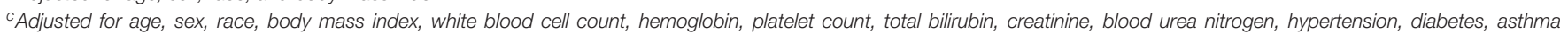
congestive heart failure, coronary heart disease, stroke, chronic bronchitis, and cancer.

TABLE 3 | Association of yogurt consumption with mortality according to age, sex, race/ethnicity, and BMI.

\begin{tabular}{|c|c|c|}
\hline Subgroup & All-cause mortality HR $(95 \% \mathrm{Cl})^{*}$ & $p$-value \\
\hline \multicolumn{3}{|l|}{ Gender } \\
\hline Male & $0.89(0.70,1.15)$ & 0.403 \\
\hline Female & $0.77(0.62,0.97)$ & 0.028 \\
\hline \multicolumn{3}{|l|}{ Age } \\
\hline Aged < 60 y & $0.96(0.65,1.40)$ & 0.851 \\
\hline Aged $\geq 60 y$ & $0.80(0.68,0.96)$ & 0.017 \\
\hline \multicolumn{3}{|l|}{ Race/Ethnicity } \\
\hline Mexican American & $0.66(0.40,1.10)$ & 0.113 \\
\hline Other Hispanic & $1.85(0.94,3.64)$ & 0.073 \\
\hline Non-Hispanic White & $0.87(0.73,1.05)$ & 0.170 \\
\hline Non-Hispanic Black & $0.54(0.30,0.97)$ & 0.042 \\
\hline Other Race & $0.40(0.13,1.21)$ & 0.107 \\
\hline \multicolumn{3}{|l|}{ Weight } \\
\hline Underweight (BMI < 18.5) & $0.44(0.16,1.18)$ & 0.104 \\
\hline Normal (BMI, 18.5 to 24.9) & $0.78(0.59,1.04)$ & 0.096 \\
\hline Overweight (BMI, 25 to 29.9) & $0.86(0.66,1.14)$ & 0.313 \\
\hline Obese (BMI $\geq 30)$ & $0.83(0.60,1.15)$ & 0.275 \\
\hline
\end{tabular}

*Adjusted for age, sex, race, body mass index, white blood cell count, hemoglobin, platelet count, total bilirubin, creatinine, blood urea nitrogen, hypertension, diabetes, asthma congestive heart failure, coronary heart disease, stroke, chronic bronchitis, and cancer.

probiotic intake might reduce enteric bacterial translocation and suppress the growth of pathogenic microbiota, indicating that probiotic could prevent enteric infections and inhibit the growth of intestinal carcinoma $(36,37)$. Furthermore, intake of probiotic significantly might improve vitamins and minerals status such as vitamin $\mathrm{K}$ and calcium which played an important role in maintaining normal physiological activities (38).

An important implication from this study was that yogurt consumption might be an important public health strategy that had been associated with decreased mortality risk among adults. Our study revealed that only $11.4 \%$ of participants had exposure to yogurt and/or dietary supplements containing probiotic among U.S. adults, which were similar to those previously reported (13.1\%) (39). It indicated that the consumption of probiotic food remained relatively unpopular and there was still much potential for improvement in US adults. Appropriate strategies were needed to encourage the wider population to accept probiotic food such as health promotion and health education around the benefits of probiotic food.

This study had several strengths. The use of a large size, nationally representative sample would improve the ability to address the impact of possible confounding and increase the generalizability and external validity of our results. In addition, we further conducted subgroup analyses according to age, gender, race/ethnicity, and BMI, and found that female, older participants (aged $\geq 60 \mathrm{y}$ ), and Non-Hispanic Black were more likely to benefit from yogurt consumption. At the same time, several limitations of this study should be recognized. First, dietary data was self-reported in the NHANES survey, which might result in measurement error inevitably. Second, due to the lack of detailed information about yogurt consumption, we could not conduct subgroup analyses to explore the dose-response association between yogurt consumption and mortality. Third, this was a retrospective, observational study, which was subject to confounding bias. Although we carefully controlled for possible confounding factors, residual confounding or unmeasured confounding could not be fully ruled out. Moreover, because dietary data was collected based on the Dietary Interview-First Day/Second Day and Dietary Supplement Use 30-Day, dietary changes could not be excluded after the survey. However, dietary changes were likely to lead to an underestimation instead of an overestimation of probiotic benefits.

\section{CONCLUSIONS}

In this study, we found that yogurt consumption was associated with a lower risk of all-cause mortality in the general population, especially among female, older participants, and Non-Hispanic Black. Yogurt consumption in diet might be a sensible strategy for reducing the risk of death. 


\section{DATA AVAILABILITY STATEMENT}

Publicly available datasets were analyzed in this study. Data used for this study are available on the NHANES website: https:// wwwn.cdc.gov/nchs/nhanes/.

\section{ETHICS STATEMENT}

The studies involving human participants were reviewed and approved by the institutional review board of the National Center for Health Statistics, CDC. The patients/participants provided their written informed consent to participate in this study.

\section{AUTHOR CONTRIBUTIONS}

PL and XG contributed to study design, data collection, data analysis, and drafting the article. ZL and TW contributed

\section{REFERENCES}

1. Sommer F, Bäckhed F. The gut microbiota-masters of host development and physiology. Nat Rev Microbiol. (2013) 11:227-38. doi: 10.1038/nrmicro2974

2. Nicholson JK, Holmes E, Kinross J, Burcelin R, Gibson G, Jia W, et al. Host-gut microbiota metabolic interactions. Science. (2012) 336:12627. doi: $10.1126 /$ science. 1223813

3. Clemente JC, Ursell LK, Parfrey LW, Knight R. The impact of the gut microbiota on human health: an integrative view. Cell. (2012) 148:125870. doi: 10.1016/j.cell.2012.01.035

4. Kamada N, Seo SU, Chen GY, Núñez G. Role of the gut microbiota in immunity and inflammatory disease. Nat Rev Immunol. (2013) 13:32135. doi: 10.1038/nri3430

5. Tang WHW, Kitai T, Hazen SL. Gut microbiota in cardiovascular health and disease. Circ Res. (2017) 120:118396. doi: 10.1161/CIRCRESAHA.117.309715

6. Fan Y, Pedersen O. Gut microbiota in human metabolic health and disease. Nat Rev Microbiol. (2021) 19:55-71. doi: 10.1038/s41579-020-0433-9

7. Cryan JF, O'Riordan KJ, Sandhu K, Peterson V, Dinan TG. The gut microbiome in neurological disorders. Lancet Neurol. (2020) 19:17994. doi: 10.1016/S1474-4422(19)30356-4

8. Korpela K, Salonen A, Vepsäläinen O, Suomalainen M, Kolmeder C, Varjosalo $\mathrm{M}$, et al. Probiotic supplementation restores normal microbiota composition and function in antibiotic-treated and in caesarean-born infants. Microbiome. (2018) 6:182. doi: 10.1186/s40168-018-0567-4

9. Quigley EMM. Prebiotics and probiotics in digestive health. Clin. Gastroenterol Hepatol. (2019) 17:333-44. doi: 10.1016/j.cgh.2018.09.028

10. Akbari E, Asemi Z, Daneshvar Kakhaki R, Bahmani F, Kouchaki E, Tamtaji OR, et al. Effect of probiotic supplementation on cognitive function and metabolic status in Alzheimer's disease: a randomized, double-blind and controlled trial. Front Aging Neurosci. (2016) 8:256. doi: 10.3389/fnagi.2016.00256

11. Ford AC, Quigley EM, Lacy BE, Lembo AJ, Saito YA, Schiller LR, et al. Efficacy of prebiotics, probiotics, and synbiotics in irritable bowel syndrome and chronic idiopathic constipation: systematic review and meta-analysis. $\mathrm{Am}$ J Gastroenterol. (2014) 109:1547-61. doi: 10.1038/ajg.2014.202

12. Malik M, Suboc TM, Tyagi S, Salzman N, Wang J, Ying R, et al. Lactobacillus plantarum 299v supplementation improves vascular endothelial function and reduces inflammatory biomarkers in men with stable coronary artery disease. Circ Res. (2018) 123:1091-102. doi: 10.1161/CIRCRESAHA.118.3 13565

13. Soleimani A, Zarrati Mojarrad M, Bahmani F, Taghizadeh M, Ramezani M, Tajabadi-Ebrahimi M, et al. Probiotic supplementation in diabetic hemodialysis patients has beneficial metabolic effects. Kidney Int. (2017) 91:435-42. doi: 10.1016/j.kint.2016.09.040 to study design, critical revision and submitted the report for publication. All authors read and approved the final manuscript.

\section{FUNDING}

This study was funded by the National Key Research and Development Program of China (Grant No. 2016YFC1304303) and the National Natural Science Foundation of China (Grant No. 31701011).

\section{SUPPLEMENTARY MATERIAL}

The Supplementary Material for this article can be found online at: https://www.frontiersin.org/articles/10.3389/fnut.2022. 803076/full\#supplementary-material

14. Khalesi S, Sun J, Buys N, Jayasinghe R. Effect of probiotics on blood pressure: a systematic review and meta-analysis of randomized, controlled trials. Hypertension. (2014) 64:897903. doi: 10.1161/HYPERTENSIONAHA.114.03469

15. Khalili L, Alipour B, Asghari Jafarabadi M, Hassanalilou T, Mesgari Abbasi M, Faraji I. Probiotic assisted weight management as a main factor for glycemic control in patients with type 2 diabetes: a randomized controlled trial. Diabetol Metab Syndr. (2019) 11:5. doi: 10.1186/s13098-019-0400-7

16. Montassier E, Valdés-Mas R, Batard E, Zmora N, Dori-Bachash M, Suez J, et al. Probiotics impact the antibiotic resistance gene reservoir along the human GI tract in a person-specific and antibiotic-dependent manner. Nat Microbiol. (2021) 6:1043-54. doi: 10.1038/s41564-021-00920-0

17. Luoto R, Ruuskanen O, Waris M, Kalliomäki M, Salminen S, Isolauri E. Prebiotic and probiotic supplementation prevents rhinovirus infections in preterm infants: a randomized, placebo-controlled trial. J Allergy Clin Immunol. (2014) 133:405-13. doi: 10.1016/j.jaci.2013.08.020

18. Khalesi S, Bellissimo N, Vandelanotte C, Williams S, Stanley D, Irwin C, et al. review of probiotic supplementation in healthy adults: helpful or hype? Eur $J$ Clin Nutr. (2019) 73:24-37. doi: 10.1038/s41430-018-0135-9

19. Centers for Disease Control and Prevention (CDC). About the National Health and Nutrition Examination Survey. (2017). Available online at: https://www. cdc.gov/nchs/nhanes/about_nhanes.htm.

20. National Center for Health Statistics. Office of Analysis and Epidemiology, Public-use Linked Mortality File. Hyattsville: Maryland (2015). Available online at:https://www.cdc.gov/nchs/data-linkage/mortality-public.htm.

21. Lourens-Hattingh A, Viljoen BC. Yogurt as probiotic carrier food. Int Dairy J. (2001) 11:1-17. doi: 10.1016/S0958-6946(01)00036-X

22. Yacoub R, Kaji D, Patel SN, Simoes PK, Busayavalasa D, Nadkarni $\mathrm{GN}$, et al. Association between probiotic and yogurt consumption and kidney disease: insights from NHANES. Nutr J. (2016) 15:1-4. 10. doi: 10.1186/s12937-016-0127-3

23. Soedamah-Muthu SS, Masset G, Verberne L, Geleijnse JM, Brunner EJ. Consumption of dairy products and associations with incident diabetes, CHD and mortality in the Whitehall II study. British J Nutr. (2013) 109:71826. doi: 10.1017/S0007114512001845

24. Praagman J, Dalmeijer GW, Van Der Schouw YT, Soedamah-Muthu SS, Verschuren WMM. Bueno-de-Mesquita HB, et al. The relationship between fermented food intake and mortality risk in the European Prospective investigation into cancer and nutrition-Netherlands cohort. British J Nutr. (2015) 113:498-506. doi: 10.1017/S0007114514003766

25. Yoon K, Kim N. Roles of Sex Hormones and Gender in the Gut Microbiota. J Neurogastroenterol Motil. (2021) 27:314-25. doi: 10.5056/jnm20208

26. Gad AS, Kholif AM, Sayed AF. Evaluation of the nutritional value of functional yogurt resulting from combination of date palm syrup and skim milk. Am J Food Technol. (2010) 5:250-9. doi: 10.3923/ajft.2010.250.259 
27. Williams EB, Hooper B, Spiro A, Stanner S. The contribution of yogurt to nutrient intakes across the life course. Nutr Bull. (2015) 40:932. doi: $10.1111 /$ nbu. 12130

28. Bakircioglu D, Topraksever N, Yurtsever S, Kizildere M, Kurtulus YB. Investigation of macro, micro and toxic element concentrations of milk and fermented milks products by using an inductively coupled plasma optical emission spectrometer, to improve food safety in Turkey. Microchemical J. (2018) 136:133-8. doi: 10.1016/j.microc.2016.10.014

29. Pontes K, Guedes MR, Cunha MRD, Mattos SS, Barreto Silva MI, Neves $\mathrm{MF}$, et al. Effects of probiotics on body adiposity and cardiovascular risk markers in individuals with overweight and obesity: a systematic review and meta-analysis of randomized controlled trials. Clin Nutr. (2021) 40:491531. doi: 10.1016/j.clnu.2021.06.023

30. Liang T, Wu L, Xi Y, Li Y, Xie X, Fan C, et al. Probiotics supplementation improves hyperglycemia, hypercholesterolemia, and hypertension in type 2 diabetes mellitus: an update of meta-analysis. Crit Rev Food Sci Nutr. (2021) 61:1670-88. doi: 10.1080/10408398.2020.1764488

31. Panahi S, Tremblay A. The potential role of yogurt in weight management and prevention of type 2 diabetes. J Am Coll Nutr. (2016) 35:71731. doi: 10.1080/07315724.2015.1102103

32. Asemi Z, Jazayeri S, Najafi M, Samimi M, Mofid V, Shidfar F, et al. Effect of daily consumption of probiotic yogurt on oxidative stress in pregnant women: a randomized controlled clinical trial. Ann Nutr Metab. (2012) 60:62-8. doi: 10.1159/000335468

33. Ejtahed HS, Mohtadi-Nia J, Homayouni-Rad A, Niafar M, Asghari-Jafarabadi M, Mofid V. Probiotic yogurt improves antioxidant status in type 2 diabetic patients. Nutr. (2012) 28:539-43. doi: 10.1016/j.nut.2011.08.013

34. Vasquez EC, Pereira T, Peotta VA, Baldo MP, Campos-Toimil M. Probiotics as beneficial dietary supplements to prevent and treat cardiovascular diseases: uncovering their impact on oxidative stress. Oxid Med Cell Longev. (2019) 2019:3086270. doi: 10.1155/2019/3086270

35. Szulińska M, Łoniewski I, Skrypnik K, Sobieska M, Korybalska K, Suliburska J, et al. Multispecies probiotic supplementation favorably affects vascular function and reduces arterial stiffness in obese postmenopausal WomenA 12-week placebo-controlled and randomized clinical study. Nutr. (2018) 10:1672. doi: 10.3390/nu10111672

36. Sato J, Kanazawa A, Azuma K, Ikeda F, Goto H, Komiya K, et al. Probiotic reduces bacterial translocation in type 2 diabetes mellitus: a randomised controlled study. Sci Rep. (2017) 7:12115. doi: 10.1038/s41598-017-12535-9

37. Perdigon G, Alvarez S, Rachid M, Agüero G, Gobbato N. Immune system stimulation by probiotics. J Dairy Sci. (1995) 78:1597-606. doi: 10.3168/jds.S0022-0302(95)76784-4

38. Knapen MHJ, Braam LA, Teunissen KJ, Zwijsen RML, Theuwissen E, Vermeer C. Yogurt drink fortified with menaquinone-7 improves vitamin $\mathrm{K}$ status in a healthy population. J Nutr Sci. (2015) 4:e35. doi: 10.1017/jns.2015.25

39. Lau E, Neves JS, Ferreira-Magalhães M, Carvalho D, Freitas P. Probiotic ingestion, obesity, and metabolic-related disorders: results from NHANES, 1999-2014. Nutr. (2019) 11:1482. doi: 10.3390/nu11071482

Conflict of Interest: The authors declare that the research was conducted in the absence of any commercial or financial relationships that could be construed as a potential conflict of interest.

Publisher's Note: All claims expressed in this article are solely those of the authors and do not necessarily represent those of their affiliated organizations, or those of the publisher, the editors and the reviewers. Any product that may be evaluated in this article, or claim that may be made by its manufacturer, is not guaranteed or endorsed by the publisher.

Copyright (c) 2022 Lin, Gui, Liang and Wang. This is an open-access article distributed under the terms of the Creative Commons Attribution License (CC BY). The use, distribution or reproduction in other forums is permitted, provided the original author(s) and the copyright owner(s) are credited and that the original publication in this journal is cited, in accordance with accepted academic practice. No use, distribution or reproduction is permitted which does not comply with these terms. 